\title{
A branch-and-bound algorithm to solve large scale integer quadratic multi-knapsack problems
}

$\underline{\text { Dominique Quadri** }}^{*}$ Eric Soutif*, Pierre Tolla**

**Lamsade, University Paris Dauphine

${ }^{*}$ Cedric, CNAM Paris

SOFSEM 2007, January 20-26 2007, Harrachov, Czech Republic 


\section{Talk overview}

e The problem

e Existing branch-and-bound

e The proposed branch-and-bound

e Computational results

e Conclusions and future works 


\section{The problem}

$(Q M K P)\left\{\begin{array}{l}\max f(x)=\sum_{i=1}^{n}\left(c_{i} x_{i}-d_{i} x_{i}^{2}\right)=\sum_{i=1}^{n} f_{i}\left(x_{i}\right) \\ \text { s.t. } \quad \begin{array}{l}\sum_{i=1}^{n} a_{j i} x_{i} \leq b_{j} \quad j=(1,2, \ldots, m) \\ 0 \leq x_{i} \leq u_{i} \quad \text { integer } \\ \text { where } \\ c_{i} \geq 0, d_{i} \geq 0, a_{j i} \geq 0, b_{j} \geq 0, u_{i} \leq\left(c_{i} / 2 d_{i}\right)\end{array}\end{array}\right.$

e We are interested in an integer quadratic multi-knapsack problem with a separable objective function.

e NP-hard problem

e Our aim : develop a practical method to solve to optimality $(Q M K P)$ 


\section{Notations}

e Let $(P)$ be a pure integer or $0-1$ program

e Let $(\bar{P})$ be the LP relaxation of $(P)$

e $Z[P]$ : optimal value of the problem $(P)$

e $Z[\bar{P}]$ : optimal value of $(\bar{P})$. 


\section{Branch-and-bound algorithm}

e A feasible solution

e A tight upper bound at each node of the search tree

e Before starting the branch-and-bound procedure : preprocessing techniques 


\section{Standard $B \& B$ approach (SBB)}

e Quadratic concave objective function subject to $m$ linear constraints

e $Z[\overline{Q M K P}]:$ upper bound

e Cplex9.0. 


\section{A 0-1 linearization $B \& B$ (LBB)}

e Transform $(Q M K P)$ into a 0-1 equivalent problem :

a direct expansion : re-write the integer variables into 0 -1 variables

e piecewise linear interpolation

e Mathur and Salkin (1983) : branch-and-bound to solve the single constraint integer quadratic knapsack $(Q K P)$ 


\section{A 0-1 linearization $B \& B$ (LBB)}

$$
\begin{gathered}
f_{i u_{i}}=c_{i} u_{i}-d_{i} u_{i}^{2} \\
f_{i k}=c_{i} k-d_{i} k^{2} \\
f_{i, k-1}=c_{i}(k-1)-d_{i}(k-1)^{2}
\end{gathered}
$$$$
f_{i 1}=c_{i} \cdot 1-d_{i} \cdot 1^{2}
$$

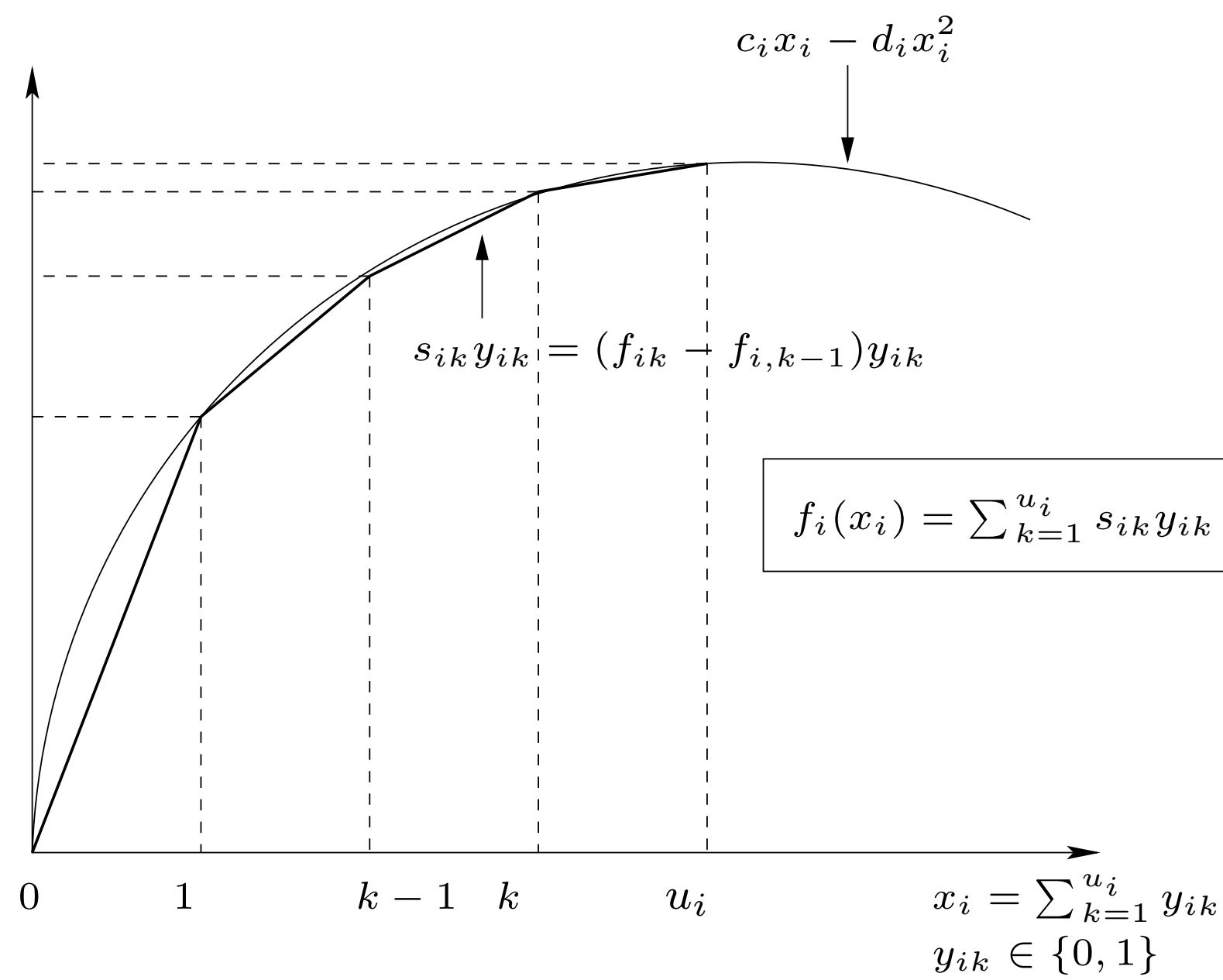




\section{A 0-1 linearization $B \& B$ (LBB)}

$$
(M K P)\left\{\begin{array}{l|l}
\max & \sum_{i=1}^{n}\left(\sum_{k=1}^{u_{i}} s_{i k} y_{i k}\right) \\
\text { s.t. } & \begin{array}{l}
\sum_{i=1}^{n}\left(a_{j i} \sum_{k=1}^{u_{i}} y_{i k}\right) \leq b_{j} \\
(j=1,2 \ldots, m) \\
y_{i k} \in\{0 ; 1\}
\end{array}
\end{array}\right.
$$

where

e $x_{i}=\sum_{k=1}^{u_{i}} y_{i k}, y_{i k} \in\{0 ; 1\}$,

e $s_{i k}=f_{i k}-f_{i, k-1}$,

e $f_{i k}=c_{i} k-d_{i} k^{2}$.

Proposition : $Z[\overline{M K P}] \leq Z[\overline{Q M K P}]$ 


\section{Djerdjour et al. algorithm UB}

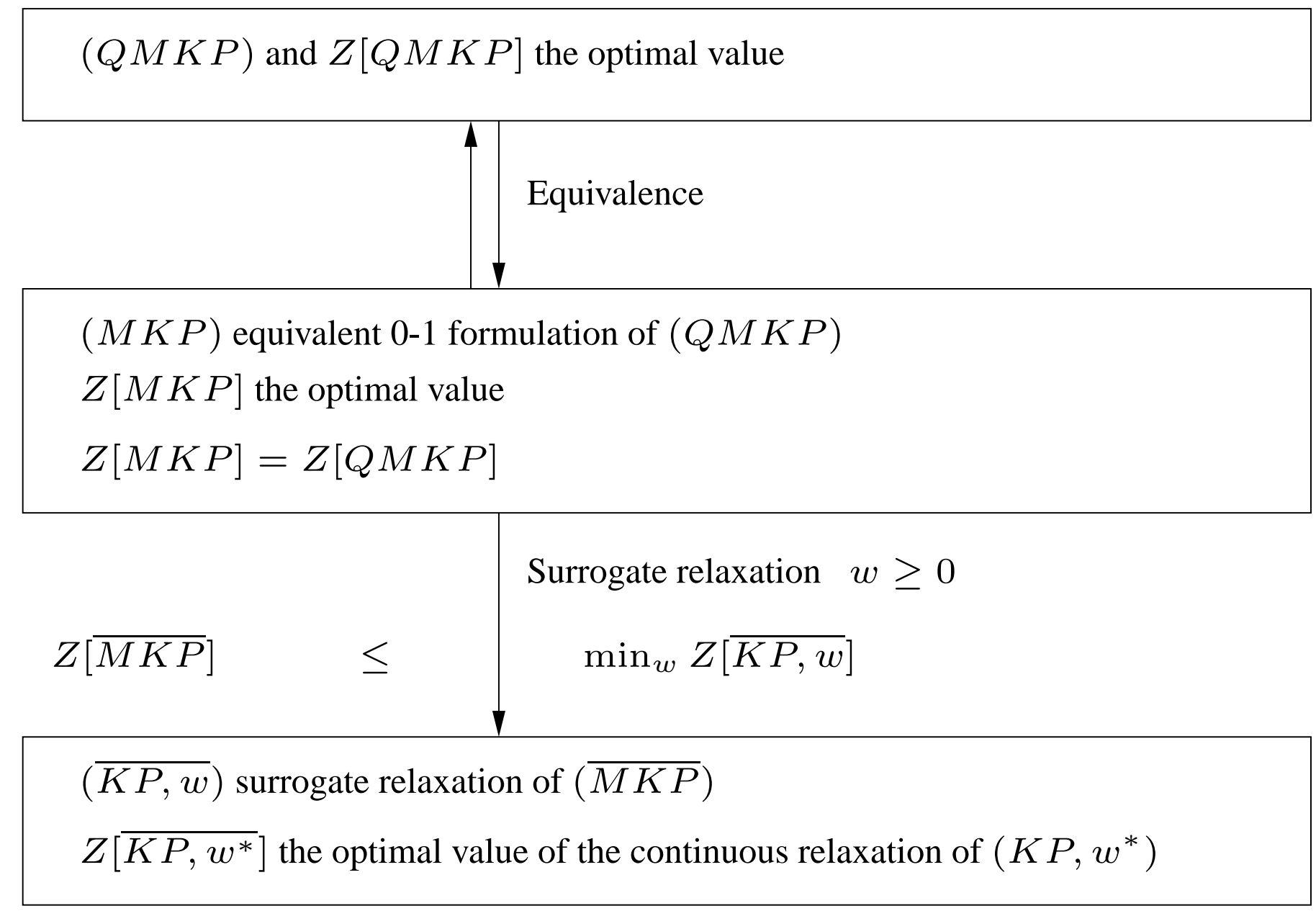




\section{Djerdjour et al. algorithm (DMS)}

e Surrogate relaxation : transform the $m$ constraints of $(\overline{M K P})$ into one constraint (called surrogate constraint);

e Surrogate multiplier : $w=\left(w_{1}, \ldots, w_{j}, \ldots, w_{m}\right) \geq 0$;

e. $(\overline{M K P})$ becomes :

$$
(\overline{K P, w})\left\{\begin{array}{l|l}
\max & \sum_{i=1}^{n}\left(\sum_{k=1}^{u_{i}} s_{i k} y_{i k}\right) \\
\text { s.t. } & \begin{array}{l}
\sum_{i=1}^{n}\left[\sum_{j=1}^{m} w_{j} a_{j i}\right] \sum_{k=1}^{u_{i}} y_{i k} \leq \sum_{j=1}^{m} w_{j} b_{j} \\
y_{i k} \in[0 ; 1]
\end{array}
\end{array}\right.
$$

e $Z[\overline{M K P}] \leq Z[\overline{K P, w}]$

e How to find a good surrogate multiplier $w^{*}$ ? 


\section{How to find $w^{*}$ ? (DMS)}

e Let us consider : $Z[\overline{K P, w}]$

e Solving $(S D)=\min _{w \geq 0} Z[\overline{K P, w}]$

e $(S D)$ is called the surrogate dual

e Problem easy to solve :

e The objective function of $(S D)$ is quasi-convexe

a Local descent method

e $w^{*}$ is a global mimimum 


\section{The proposed $B \& B$}

e Improving the upper bound of (DMS)

a Decreasing the computational time

a Getting a tighter upper bound

e A heuristic to compute a feasible solution

e Pre-processing procedures 


\section{Decreasing the computational time}

e Proposition 1

If $w^{*}$ is the dual optimal solution of $(\overline{M K P})$ then the optimal value of $(\overline{M K P})$ is equal to the optimal value of $\left(\overline{K P, w^{*}}\right)$ that is :

$Z[\overline{M K P}]=Z\left[\overline{K P, w^{*}}\right]$

e Decreasing the computational time of $w^{*}$

e $w^{*}$ : dual optimal solution of $(\overline{M K P})$ 


\section{Getting a tighter upper bound}

e Improving the upper bound value

e $Z\left[K P, w^{*}\right]$ : an improved upper bound

e Analytically the upper bound is improved. 


\section{Analytical comparison of the upper bounds}

\begin{tabular}{|c|c|c|}
\hline Problem & $+\infty$ & Upper bound \\
\hline \multirow[t]{3}{*}{$(\overline{Q M K P})$} & & $Z[\overline{Q M K P}]$ \\
\hline & & [LP relaxation] \\
\hline & & [Djerdjour at al. 1988] \\
\hline$\left(\overline{K P, w^{*}}\right)$ & & $Z\left[\overline{K P, w^{*}}\right]$ \\
\hline$(\overline{M K P})$ & & $Z[\overline{M K P}]$ \\
\hline & & [Linearized formulation] \\
\hline$\left(K P, w^{*}\right)$ & & $Z\left[K P, w^{*}\right]$ \\
\hline & & [Our approach] \\
\hline$(M K P)$ & & Linearized optimum \\
\hline $\mathbb{1}$ & & $=$ \\
\hline$(Q M K P)$ & & Quadratic optimum \\
\hline
\end{tabular}




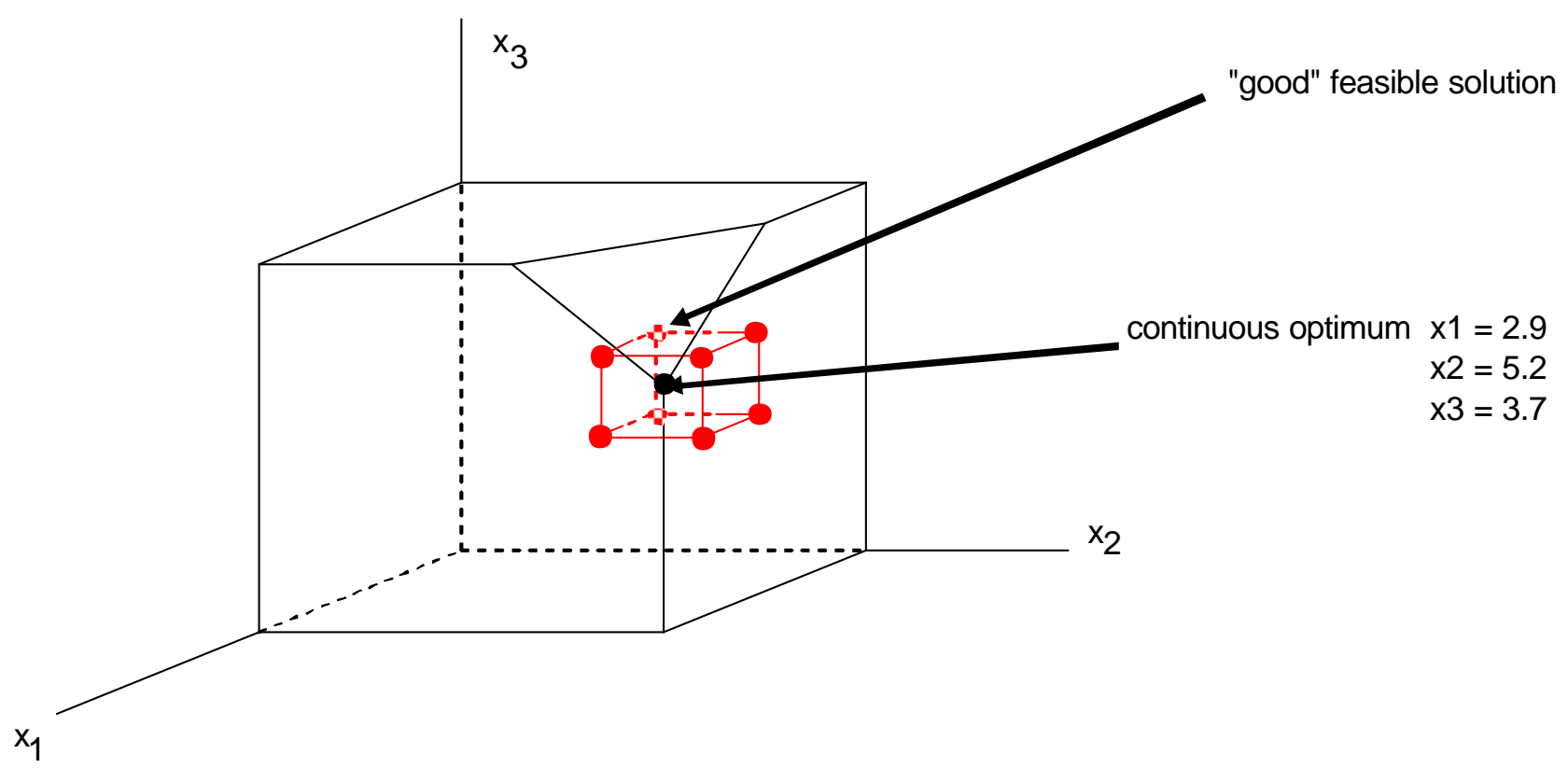




\section{Pre-processing procedures}

e Detecting some redundant constraints

e Reducing the bounds of integer variables: contraints pairing procedure, Hammer et al. (1975).

e Simultaneously fixing some $0-1$ variables to 0 


\section{Computational results}

e square problems $(n=m)$

e problems are randomly generated in the interval $[0,100]$ according to an uniform law

e average $\%$ of pure integer variables : $40 \%$ for squared problems

e average value of $u_{i}: 22$ for squared problems. 


\section{Average CPU time of the $4 B \& B$}

\begin{tabular}{llcccc}
\hline$n$ & $m$ & Our BB & $L B B$ & $S B B$ & $D M S$ \\
\hline 100 & 100 & 1.5 & 1.3 & 7.8 & 208.257 \\
500 & 500 & 29.3 & 120.1 & 19.1 & - \\
1000 & 1000 & 50.5 & 264.4 & 282.3 & - \\
1500 & 1500 & 183.7 & 392.5 & 1178.4 & - \\
2000 & 2000 & 305.2 & 1369.4 & 2557.9 & - \\
\hline
\end{tabular}

“-" : optimum not reached in a limit time of 3 hours 


\section{Analyzing the computational results}

The improvement capability of our $B \& B$ can be explained by three features, namely:

1. the feasible solution

2. the upper bound

3. the pre-processing procedures 


\section{The upper bound}

\begin{tabular}{llcccccccc}
\hline \multicolumn{4}{c}{ Av. deviation to the opt. (\%) } & \multicolumn{5}{c}{ CPU time (sec.) } \\
& \multicolumn{3}{c}{ Our BB } & LBB=DMS & SBB & Our BB & LBB & SBB & DMS \\
100 & 100 & 8.2 & 9.5 & 16.9 & 0.0 & 0.0 & 0.0 & 0.3 \\
500 & 500 & 7.5 & 7.9 & 12.9 & 0.2 & 0.1 & 7.3 & 9.0 \\
1000 & 1000 & 21.7 & 23.0 & 32.2 & 0.5 & 0.5 & 58.2 & 37.9 \\
1500 & 1500 & 23.9 & 24.6 & 37.8 & 1.6 & 1.5 & 184.5 & 86.6 \\
2000 & 2000 & 36.2 & 36.9 & 53.0 & 3.6 & 3.4 & 421.3 & 157.8 \\
\hline
\end{tabular}




\section{The pre-processing procedures}

e Detecting some redundant constraints : on average $52 \%$ of the constraints may be removed

e Reducing the bounds of integer variables : the average proportion of pure integer variables has decreased from $40 \%$ to $21.02 \%$

e Simultaneously fixing some $0-1$ variables to 0 : $50.25 \%$ of $0-1$ variables are fixed 


\section{Conclusions and future works}

e Conclusions

e Our $B \& B$ allowed us to solve large scale instances : up to 2000 variables within 306 s on average (largest problems)

e (LBB) is a possible alternative to solve ( $Q M K P)$

e (SBB) and (DMS) can be used only for small instances

e Future works

e Improve our upper bound

a Solve a nonseparable quadratic multi-knapsack problem 